Article

\title{
Downregulation of the Polycomb-Associated Methyltransferase Ezh2 during Maturation of Hippocampal Neurons Is Mediated by MicroRNAs Let-7 and miR-124
}

\author{
Laura Guajardo 1,2, Rodrigo Aguilar 1,2(D), Fernando J. Bustos ${ }^{1,2,3}$, Gino Nardocci ${ }^{1,2}$ (D), \\ Rodrigo A. Gutiérrez ${ }^{2,4}\left(\mathbb{0}\right.$, Brigitte van Zundert ${ }^{1,3, *}$ and Martin Montecino ${ }^{1,2, *}$ \\ 1 Institute of Biomedical Sciences, Faculty of Medicine and Faculty of Life Sciences, Universidad Andres Bello, \\ Santiago 8370186, Chile; lau.guajardoc@gmail.com (L.G.); rodrigo.aguilar@unab.cl (R.A.); \\ fernando.bustos@unab.cl (F.J.B.); gnardocci@uandes.cl (G.N.) \\ 2 FONDAP Center for Genome Regulation, Santiago 8370186, Chile; rgutierrez@bio.puc.cl \\ 3 CARE Biomedical Research Center, Santiago 83370186, Chile \\ 4 Millennium Institute for Integrative Biology, Departamento de Genética Molecular y Microbiología, \\ Facultad de Ciencias Biológicas, Pontificia Universidad Católica de Chile, Santiago 8331150, Chile \\ * Correspondence: bvanzundert@unab.cl (B.v.Z.); mmontecino@unab.cl (M.M.)
}

Received: 6 October 2020; Accepted: 6 November 2020; Published: 11 November 2020

\begin{abstract}
Ezh2 is a catalytic subunit of the polycomb repressive complex 2 (PRC2) which mediates epigenetic gene silencing through depositing the mark histone $\mathrm{H} 3$ lysine 27 trimethylation (H3K27me3) at target genomic sequences. Previous studies have demonstrated that Enhancer of Zeste Homolog 2 (Ezh2) was differentially expressed during maturation of hippocampal neurons; in immature neurons, Ezh2 was abundantly expressed, whereas in mature neurons the expression Ezh2 was significantly reduced. Here, we report that Ezh2 is downregulated by microRNAs (miRs) that are expressed during the hippocampal maturation process. We show that, in mature hippocampal neurons, lethal-7 (let-7) and microRNA-124 (miR-124) are robustly expressed and can target cognate motifs at the $3^{\prime}$-UTR of the Ezh2 gene sequence to downregulate Ezh2 expression. Together, these data demonstrate that the PRC2 repressive activity during hippocampal maturation is controlled through a post-transcriptional mechanism that mediates Ezh2 downregulation in mature neurons.
\end{abstract}

Keywords: Ezh2 expression; microRNA-mediated control; let-7 and mir-124 in hippocampus

\section{Introduction}

Several reports have demonstrated the critical role of epigenetic mechanisms during control of gene expression associated with the physiological function of the central nervous system (CNS) in mammals [1,2]. These mechanisms include DNA methylation, histone post-translational modifications, and long and short non-coding RNA activities, among others [3]. A relevant component of epigenetic control in the CNS is mediated by the polycomb repressive complex 2 (PRC2), which catalyzes the deposit of the repressive histone mark trimethyl lysine 27 of histone $\mathrm{H} 3$ (H3K27me3) at target gene promoters, thereby producing their transcriptional silencing [4,5]. Our team showed that, during early stages of hippocampal neuron maturation, PRC2 activity was critical to control the expression of non-neuronal gene programs (e.g., osteogenic lineage genes), as well as the expression of key components of the dendritic arbor formation that were required at more advanced stages of maturation [6,7]. These data demonstrated that the catalytic subunit of PRC2, the methyltransferase Enhancer of Zeste Homolog 2 (Ezh2), played a key role during this gene expression silencing events in 
the hippocampus. Additionally, Ezh2 has been found expressed in neuronal progenitor cells and in neurons during early embryonic stages, where Ezh2 trimethylates H3K27 target regulatory sequences for controlling the balance between self-renewal and lineage commitment $[8,9]$.

It has been determined that, in rat hippocampal neurons, the expression of Ezh2 is downregulated during development [7]. Thus, Ezh2 is significantly expressed in the hippocampus at embryonic and early postnatal stages, where, as part of PRC2, it binds to target promoters to mediate gene silencing. At later adult stages, Ezh2 mRNA and protein are largely undetectable in hippocampal neurons and Ezh2 function in the PRC2 complex can be partially replaced by Ezh1 [6,7]. Although our results indicated that an important component mediating this Ezh2 downregulation during hippocampal maturation could involve transcriptional inhibition of the Ezh2 gene, additional evidence has demonstrated the contribution of post-transcriptional and post-translational mechanisms, including the role of microRNAs [10] and ubiquitin-dependent proteasome degradation [11].

Accumulating evidence indicates that microRNAs (miRs) are key modulators of gene expression in the CNS [12,13], where approximately $50 \%$ of the total known mammalian miRs are expressed [14,15], regulating critical processes that include dendritogenesis [16] and synapse maturation [17]. Studies have shown that conditional knockout of Dicer in mice, the endoribonuclease that mediates a critical step during miR maturation, drastically affected survival of neural stem cells and decreased differentiation of newborn neurons [18-20]. Among the microRNAs (miRs), miR-124 has been reported to play a relevant role in neuronal function, which has been found to be upregulated during neural differentiation [14,21,22]. Forced expression of miR-124 in embryonal carcinoma P19 cells in the presence of retinoic acid promotes a neuronal-like phenotype [20,22,23]. Moreover, an increased expression of miR-124 mediates the conversion of fibroblasts to functional neurons [24,25]. Studies in vivo have further shown that miR-124 was an important regulator of adult neurogenesis in mice [26]. Conversely, knockout of the miR-124-1 gene in mice resulted in defective neuronal survival, impaired hippocampal axonal outgrowth, and reduced brain size [27]. Interestingly, it was been shown that miR-124 targeted the Ezh2 3'-UTR in early neuronal cells, thereby controlling Ezh2 expression and Ezh2-mediated gene silencing during neuron lineage commitment [28]. Additionally, miR-124 targeted and downregulated USP14, coding for a deubiquitinase that binds to Ezh2, and thus prevented ubiquitin-dependent degradation of Ezh2 via proteasome [29,30].

Another relevant microRNA molecule during brain development is let-7 (lethal-7), which has been found in elevated concentrations in this tissue during embryogenesis [31-34]. Mammals contain a let-7 family, including several members (from let-7a to let-7k), which have been shown to play a significant role in maintaining the balance between neuronal progenitor cell proliferation and commitment to engage neurogenic differentiation, as they control the expression of a number of genes that are critical during this early developmental stages [35-38]. Interestingly, it has been shown that let-7 mediated control of Ezh2 gene expression in prostate cancer cells [39], as it recognized a specific motif at the Ezh2 3'-UTR. Loss of let-7 function in these tumor cells resulted in increased Ezh2 expression that was accompanied by acquisition of a cancer stem cell signature.

Whereas these data provide support for a regulatory role of miRs over PRC2 expression and function during early neuron lineage commitment, there is a lack of studies that address the contribution of miRs during the maturation of hippocampal neurons, a process that determines the functionality of these cells during learning and memory. Here, we examine whether specific miR molecules contribute to Ezh2 downregulation during hippocampal neuronal maturation. We report that miR-124 and let-7, which are among the group of miRs that are highly expressed in mature hippocampal neurons, can target cognate motifs at the Ezh2 3'-UTR, and thereby dampen Ezh2 expression. 


\section{Results}

\subsection{MicroRNA Expression Profile in Mature Hippocampal Neurons}

Previous results have indicated that expression of the methylase Ezh2, the catalytic subunit of the PRC2 complex, was downregulated during hippocampal neuronal maturation [7]. However, the mechanisms that control this reduced expression of Ezh2 (see Figure 1) have not been elucidated.

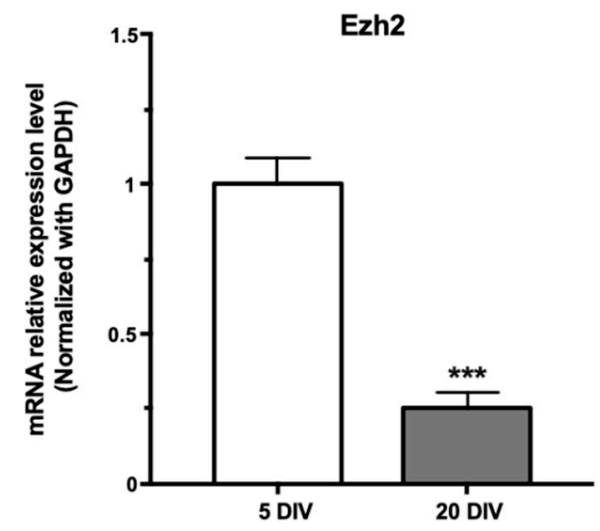

Figure 1. Expression of Enhancer of Zeste Homolog 2 (Ezh2) mRNA is downregulated during hippocampal neuronal maturation. mRNA samples were collected from hippocampal neuron-enriched cultures at 5 and 20 days in vitro (DIV). Then, real-time quantitative PCR (RT-qPCR) analysis was performed to determine the Ezh2 mRNA levels. All values are presented as relative expression normalized to Glyceraldehyde-3-Phosphate Dehydrogenase (GAPDH). $\left(n=3{ }^{* * *} p<0.001\right)$.

Accumulating evidence demonstrates that miRs are critical regulators of gene expression in the CNS, controlling key processes that include dendritogenesis and synapse maturation, among others [38]. Hence, we examined the miR expression profile in mature rat hippocampal neurons seeking to identify miR molecules that could mediate this Ezh2 mRNA downregulation (Figure 1). The miRs were isolated from neuron-enriched cell populations obtained from 18-day-old rat embryos (E18) and grown in culture for up to 20 days in vitro (20 DIV), under conditions that significantly reduced glia proliferation and favored neuron maturation $[7,40]$. The miR populations were harvested from mature (20 DIV) cell cultures, purified, properly labeled, and then analyzed using microarrays (Affimetrix Chip System). The miR expression profile data (see additional data file in Table S1) were analyzed bioinformatically to determine which miR molecules were highly expressed in these mature hippocampal neurons. Importantly, it was detected that, among the top 25 highly expressed miRs (Table 1), there were two families, i.e., miR-124 and let-7, which could potentially recognize the $3^{\prime}$-UTR end of the Ezh2 mRNA in these cells (see below), and therefore potentially contributed to downregulate Ezh2 expression during hippocampal maturation (Figure 1) [7].

Table 1. List of top 25 highly expressed microRNAs in mature hippocampal neurons. The data was originated by microarray analyses from three independent experiments $(n=1,2$, and 3$)$.

\begin{tabular}{ccccc}
\hline ProbeSetID & 20 DIV n1 & 20 DIV n2 & 20 DIV n3 & Mean 20 DIV \\
\hline rno-miR-125b-5p_st & 14,19586735 & 14,11524634 & 14,02201945 & 14,1110444 \\
rno-let-7c_st & 14,05654371 & 13,7811261 & 14,33908404 & 14,058918 \\
rno-miR-124_st & 13,14528831 & 14,67637917 & 13,74687013 & 13,8561792 \\
rno-let-7b_st & 13,77215925 & 13,48533913 & 14,02162067 & 13,7597064 \\
hp_rno-mir-124-3_s_st & 12,66468198 & 14,17408833 & 13,4111353 & 13,4166352 \\
hp_rno-mir-124-1_s_st & 12,55263545 & 14,12533502 & 13,40486244 & 13,3609443 \\
hp_rno-mir-124-2_s_st & 12,50969099 & 13,9984208 & 13,40293019 & 13,3036807 \\
\hline
\end{tabular}


Table 1. Cont.

\begin{tabular}{ccccc}
\hline ProbeSetID & 20 DIV n1 & 20 DIV n2 & 20 DIV n3 & Mean 20 DIV \\
\hline rno-miR-103_st & 12,86020989 & 13,58644686 & 13,02428652 & 13,1569811 \\
rno-miR-99a_st & 13,4265623 & 13,03790836 & 12,6824616 & 13,0489774 \\
rno-miR-107_st & 12,54827077 & 13,33459961 & 12,92074488 & 12,9345384 \\
rno-miR-16_st & 13,06704109 & 13,08511435 & 12,45829264 & 12,8701494 \\
rno-miR-138_st & 11,97407534 & 13,46384005 & 13,02131292 & 12,8197428 \\
rno-miR-24_st & 13,22507587 & 12,5403456 & 12,45652393 & 12,7406485 \\
rno-miR-22_st & 12,80712275 & 12,92439752 & 12,4873622 & 12,7396275 \\
rno-let-7a_st & 12,7448344 & 12,65429313 & 12,66395779 & 12,6876951 \\
rno-miR-181a_st & 12,18081162 & 13,05708698 & 12,71542614 & 12,6511082 \\
rno-miR-191_st & 12,97155739 & 12,54972548 & 12,42775979 & 12,6496809 \\
rno-miR-23a_st & 13,4611495 & 12,41864159 & 12,06288726 & 12,6475594 \\
rno-let-7d_st & 12,33069725 & 12,736612 & 12,86714228 & 12,6448172 \\
rno-miR-26a_st & 12,50207374 & 12,94821494 & 12,21461208 & 12,5549669 \\
rno-let-7e_st & 12,13864311 & 12,47117626 & 12,48884926 & 12,3662229 \\
rno-miR-125a-5p_st & 12,67967 & 12,30327676 & 11,86511822 & 12,2826883 \\
rno-miR-130a_st & 12,38279452 & 12,62256525 & 11,75596603 & 12,2537753 \\
rno-miR-127_st & 11,38137317 & 12,79650642 & 12,28065679 & 12,1528455 \\
rno-miR-99b_st & 12,31702088 & 11,70084872 & 11,9723612 & 11,9967436 \\
\hline
\end{tabular}

\subsection{Downregulation of Ezh2 Expression during Hippocampal Maturation Requires Dicer}

To begin examining the role of miRs on Ezh2 expression in hippocampal neurons, we carried out a knockdown of Dicer expression within these cells, the RNA nuclease that catalyzes the processing of immature miR precursors, thereby allowing the formation of mature and functional miR molecules [41,42]. Two shRNAs coding sequences targeting Dicer mRNA (shDicer1 and shDicer2) were produced as lentiviral vectors, and then infective viral particles were generated. These particles efficiently infected hippocampus neuronal cell types, including the mouse N2A neuroblastoma cell line and primary rat hippocampal neurons, where a significant decrease in Dicer mRNA expression was detected (Figure S1).

Next, we infected mature (from 17 DIV to 20 DIV) hippocampal neuron-enriched cultures with lentiviruses encoding for the two shRNAs against Dicer. It was found that both molecules downregulated Dicer mRNA levels as compared with an infection with lentiviruses encoding only GFP (Figure 2A, B, respectively). Importantly, we determined that this shRNA-mediated knockdown of Dicer expression (using a mix of both shDicer 1 and 2, Figure 2C) resulted in a significant increase in Ezh2 mRNA levels (Figure 2D). Together, these results indicate that the decreased Ezh2 expression during maturation of hippocampal neurons (Figure 1) can be mediated by miRs that require Dicer for adequate processing and function.

\subsection{Candidate miRs for Mediating Ezh2 Downregulation Exhibit Increased Expression during Hippocampal Neuronal Maturation}

Our highly expressed miR profile in mature hippocampal neurons (Table 1) revealed the presence of miRs that could potentially target the $3^{\prime}$-UTR of the Ezh2 mRNA (see Table 2). Among them, miR-124 has been previously shown to target and downregulate Ezh2 mRNA expression during early mouse neurogenesis [28] and let-7, which has been previously associated with Ezh2 mRNA control in prostate cancer cells [39]. Both miR families could recognize non-overlapping motifs at the Ezh2 $3^{\prime}$-UTR (Figure 3A) and were confirmed as upregulated during hippocampal neuronal maturation by using Taqman-based quantitative PCR approaches (see Methods) (Figure 3B). We additionally identified other miRs that could potentially target the Ezh2 3'-UTR. For instance, miR-33 (Figure 3A), although it is detected at relatively lower levels than miR-124 and let-7 members in mature hippocampal neurons (see Table S1), shows an expression that increases during the transition from immature to mature neurons (Figure 3B). It has been shown that miR-33 played a principal role during control of the 
cholesterol metabolism as it targeted and downregulated the expression of genes associated with the biosynthesis and transport of cholesterol in the liver [43]. Importantly, miR-33 has not been found to be associated with Ezh2 expression control or to play a relevant role in hippocampal neurons.

A

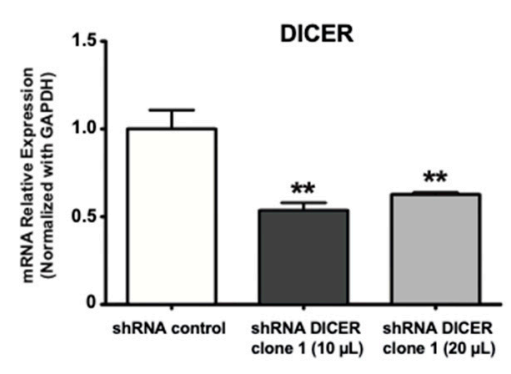

C

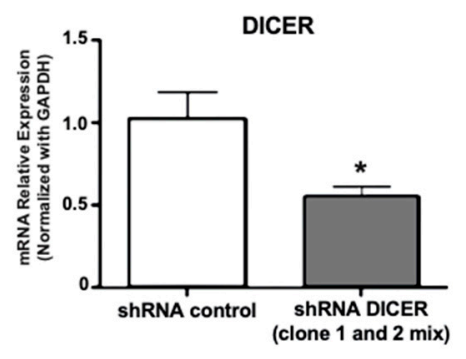

B

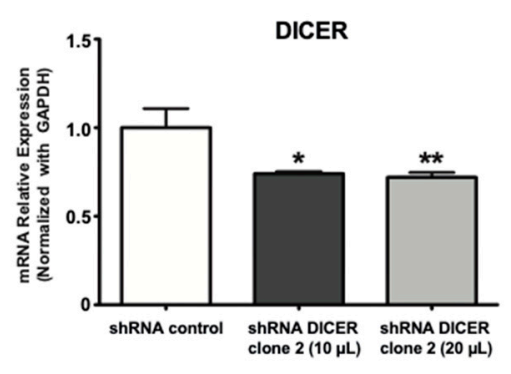

D

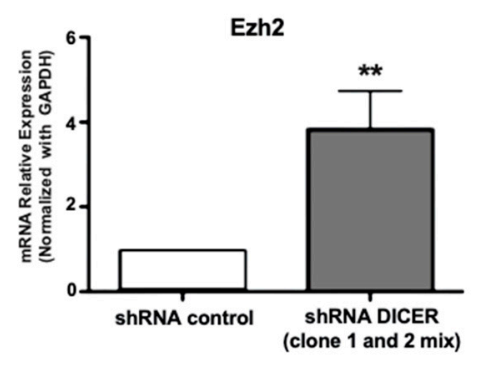

Figure 2. Dicer knockdown in primary mature hippocampal neurons results in enhanced Ezh2 mRNA expression. Reduced Dicer mRNA expression is found after lentiviral transduction of shDicer 1 (A) and 2 (B) molecules; (C) Hippocampal neuron-enriched cultures at 17 DIV were transduced for $72 \mathrm{~h}$ with a mix of lentivirus coding shRNA 1 and shRNA 2 against Dicer, and Dicer mRNA expression then analyzed; (D) Ezh2 mRNA expression in mature hippocampal neurons (20 DIV) infected with shRNA against Dicer. Results are shown as mean $\pm \operatorname{SEM}\left(n=3,{ }^{*} p<0.05\right.$ and $\left.{ }^{* *} p<0.01\right)$.

Table 2. The microRNA (miR) families that can potentially target the $3^{\prime}$-UTR region of Ezh2 mRNAs in mature rat hippocampal neuron-enriched cultures (20 DIV). Information about the specific chromosome location of each miR family member is provided. The position of miRs within a sequence that clusters additional microRNA- and other-coding genes is also indicated. IR, intergenic region.

\begin{tabular}{cccc}
\hline Target & microRNA & Chromosome & Coding Details \\
\hline & let-7a-1 & 17 & Clustered miR-3596b, miR-3596d, let-7d; let-7f-1 \\
let-7a-2 & 8 & Clustered miR-100, miR-3695a, in lnc215 gene \\
let-7b & 7 & Clustered let-7c-2 \\
let-7c-1 & 11 & Clustered miR-99a \\
let-7c-2 & 7 & Clustered let-7b \\
& let-7d & 17 & Clustered miR-3596b, miR-3596d, let-7a-1, let-7f-1, in \\
& let-7e & 1 & Spaca6 gene \\
Ezh2 & let-7f-1 & 17 & Clustered miR-99b, miR-3596c, miR-125a \\
& let-7f-2 & $X$ & Clustered 3596b, miR-3596d, let-7a-1, let-7d \\
& let-7g & 8 & Clustered miR-98, in Huwe1 gene \\
& let-7i & 7 & IR \\
miR-124-1 & 15 & IR \\
miR-124-2 & 2 & IR \\
miR-124-3 & 3 & IR \\
miR-33 & 7 & IR \\
& & & In Srebf2 gene \\
\hline
\end{tabular}




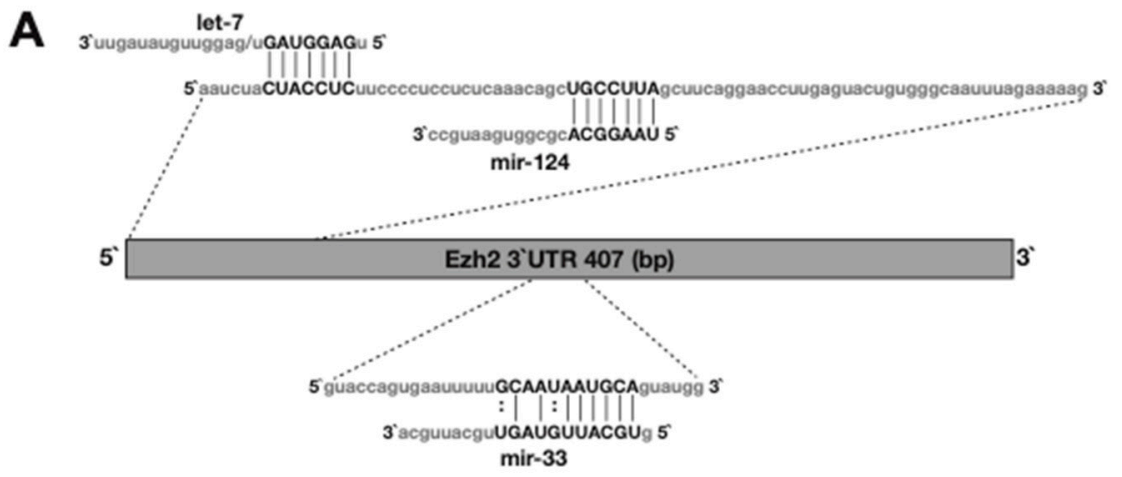

B
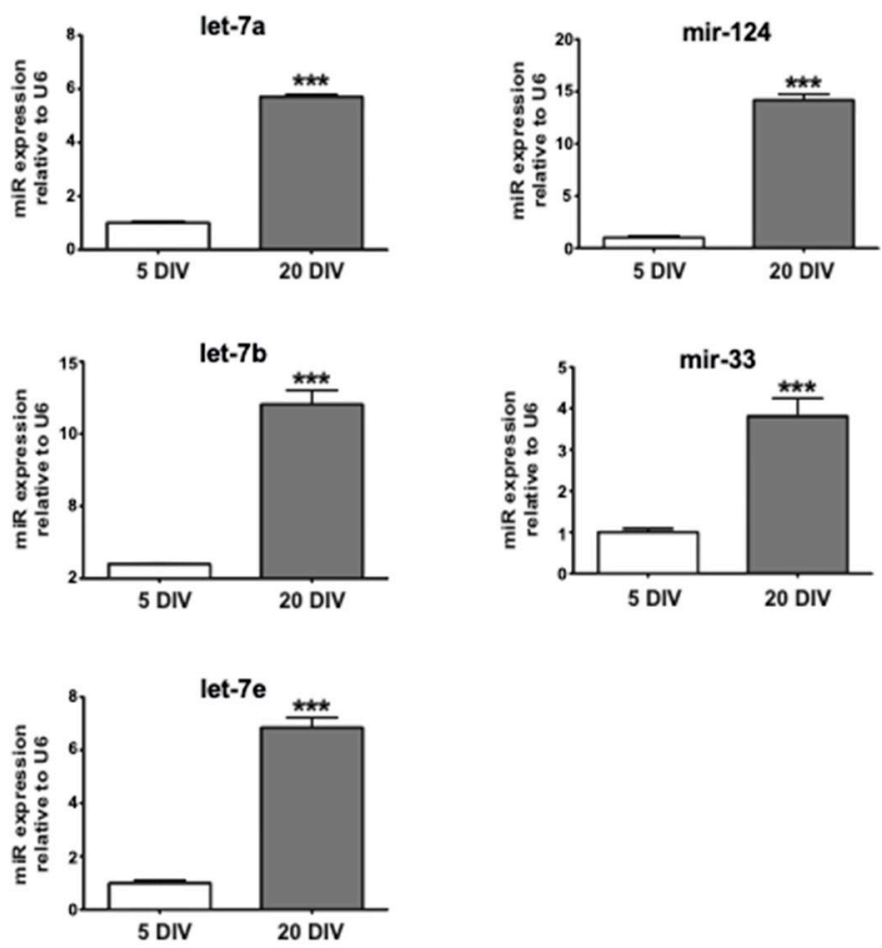

Figure 3. Expression of miRs that potentially bind at the $3^{\prime}$-UTR of Ezh2 mRNAs is upregulated during hippocampal neuronal maturation. (A) Schematic representation of Ezh2 3'-UTR and the predicted miR-124, let-7, and miR-33 target sites; (B) Quantification of miR-124, let-7 (let-7a, -b, and -e family members) and miR-33 increased expression during hippocampal neuronal maturation (5 to 20 DIV). Each miRNA was quantified using specific TaqMan ${ }^{\circledR}$ probes. Expression of the different miRNAs was normalized against snU6 RNA. Results are shown as mean \pm SEM. $\left(n=3,{ }^{* * *} p<0.001\right)$.

2.4. The 3'-UTR of the Ezh2 Gene Contains Sequence Elements that Mediate Decreased Expression of Immediately Upstream Protein-Coding Genes

Next, we examined the ability of the Ezh2 3'-UTR sequence to modulate the expression of an immediately upstream luciferase reporter gene in cells enriched in miRs that can potentially target motifs in this 3'-UTR. For this purpose, the entire Ezh2 3'-UTR segment (407 bp) was cloned downstream of the luciferase reporter gene within the context of Cytomegalovirus (CMV)-driven mammalian expression vector (CMV-LUC-Ezh2-3'UTR, see Figure 4A). This vector was transfected in both immature (5 DIV) and mature (20 DIV) hippocampal neuronal cultures for $24 \mathrm{~h}$, after which the luciferase activity present in the transfected cells was measured. It was found that transfection of this construct in mature neurons resulted in a significantly reduced luciferase activity as compared with that obtained in immature hippocampal cells (Figure 4B). This result indicates that the $3^{\prime}$-UTR of the 
Ezh2 gene can mediate a decreased biosynthesis of the luciferase protein in mature hippocampal cells that exhibit increased expression of miRs that may potentially target this $3^{\prime}$-UTR sequence.
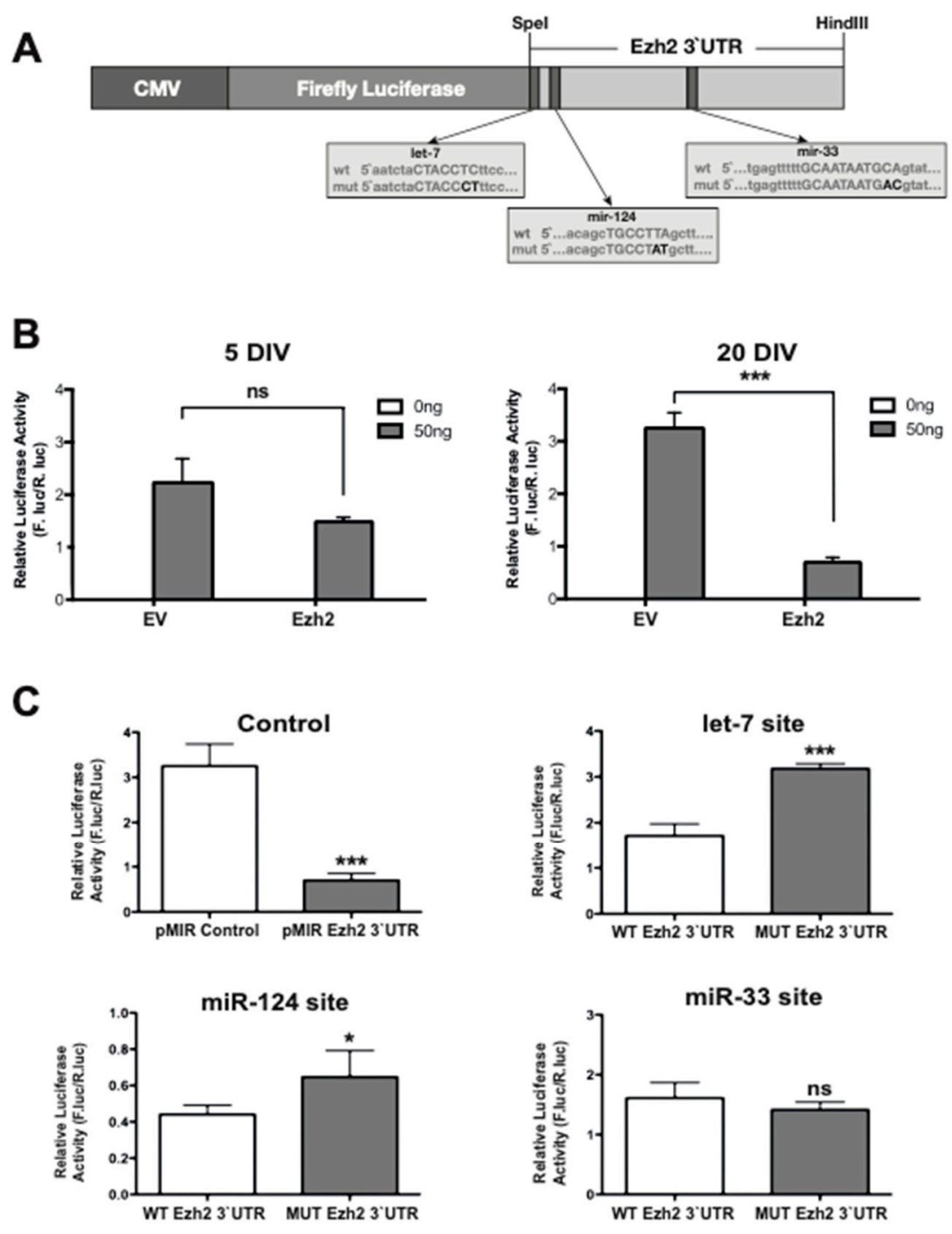

Figure 4. 3'-UTR sequence of the Ezh2 gene contains miR motifs that can downregulate the expression of an immediately up-stream luciferase reporter gene in rat hippocampal neurons. (A) Schematic representation of the CMV(Cytomegalovirus)-driven luciferase Ezh2 3'-UTR reporter gene (pMIR-report-Ezh2-3'UTR) construct expressed in hippocampal neurons following transient transfection. Wild-type (wt) and mutated (mut) motifs for miR-124, let-7, and miR-33 are shown below the construct. The specific mutations introduced are indicated in black-bold; (B) Immature (5 DIV) and mature (20 DIV) hippocampal neurons were transfected with 50 ng of empty vector (EV) or pMIR-report-Ezh2-3'UTR vector and luciferase activity measured $24 \mathrm{~h}$ later; (C) Relative luciferase activity after expression of wild-type (control) and mutant (indicated at the top of each graph) versions of let-7, miR-124, and miR-33 motifs in mature (20 DIV) hippocampal neurons. Results are shown as mean $\pm \operatorname{SEM}\left(n=3,{ }^{*} p<0.05\right.$ and $\left.{ }^{* * *} p<0.001\right)$.

Next, we examined whether the specific motifs for let-7, miR-124, and miR-33 within the $3^{\prime}$-UTR of the Ezh2 gene were contributing to mediate this downregulation of luciferase expression in mature hippocampal neurons. Specific mutations at each motif were introduced within the context of the entire Ezh2 3'-UTR sequence cloned immediately downstream of the luciferase reporter gene (CMV-LUC-Ezh2-3'UTR vector) (Figure 4A). Each mutated construct was transiently transfected in 
mature hippocampal neurons (in parallel with the wild-type version of this expression vector) and the effect on the luciferase activity was measured $24 \mathrm{~h}$ later. We determined that mutation of the cognate motifs for let-7 and miR-124 prevented the reduced luciferase activity observed with the wild-type version of the construct (Figure 4C). Thus, instead of the decreased luciferase activity (fiveto six-fold) produced by the WT Ezh2 3'-UTR construct (control graph), the mutation at the let-7 (let-7 site graph) and miR-124 (miR-124 site graph) elements resulted in higher reporter activity with respect to this wild-type construct (two- and 1.5-fold, respectively). These results suggest that joint or independent binding of endogenous let-7 and miR-124 family members to the Ezh2 3'-UTR sequence can mediate downregulation of the luciferase reporter activity. In addition, mutation of the miR-33 motif resulted in luciferase activity that was comparable to that produced by the wild-type version of the vector (Figure 4C). These results suggest that let-7 and miR-124 motifs, but not the miR-33 motif, can significantly contribute to the Ezh2 3'-UTR-dependent downregulation of Ezh2 expression in mature hippocampal neurons. It is necessary to consider that because mutation of the let-7 and miR-124 motifs did not completely revert the Ezh2 3'-UTR-mediated reduction in the luciferase reporter activity, there could be additional regulatory mechanisms associated with this $3^{\prime}$-UTR sequence that contributed to its inhibitory activity.

\subsection{Expression of a Let-7 Mimic in Immature Hippocampal Neurons Inhibits Ezh2 Expression}

To further demonstrate that miRs enriched in mature hippocampal neurons can mediate downregulation of Ezh2 expression, we performed a gain-of-function experiment using a miR mimics-based strategy $[44,45]$. Because the mutation of the let-7 motif produced the highest effect on the inhibitory activity of the Ezh2 3'-UTR sequence (Figure 4C), we examined the impact of an enhanced expression of let-7e mimics on the levels of the endogenous Ezh2 mRNA in immature (5 DIV) hippocampal neurons. As shown in Figure 5, transfection of these immature neurons with a let-7e mimic results in a robust increase in its expression as compared with a negative control mimic (termed mimic control) (Figure 5A). This let-7 increase is accompanied by a significant reduction in Ezh2 mRNA levels (Figure 5B), further indicating that Ezh2 expression is controlled by miRs that, like let-7, can target the Ezh2 3'-UTR in hippocampal neurons. Importantly, this reduced Ezh2 mRNA expression occurs concomitantly with an increase in PSD95/Dlg4 gene transcription (Figure 5B), a previously reported gene target of the PRC2-Ezh2 complex in immature hippocampal neurons [7].

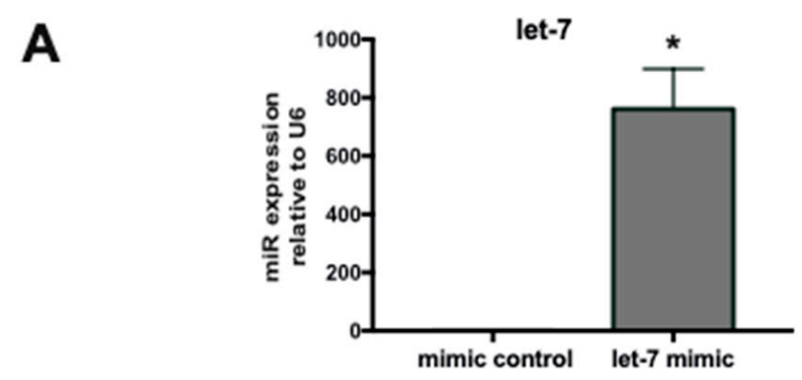

Figure 5. Cont. 


\section{B}

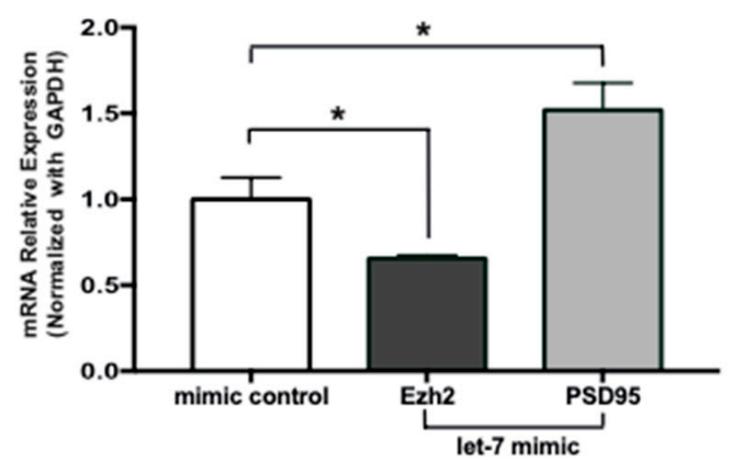

Figure 5. Forced expression of a let-7 mimic downregulates Ezh2 mRNA expression in immature hippocampal neurons. (A) Expression of let-7 was significantly higher in immature hippocampal neurons (5 DIV) following a let-7 mimic transfection for $48 \mathrm{~h}$. Quantification was assessed by TaqMan ${ }^{\circledR}$ probes as shown in Figure 3 legend; (B) Significantly decreased expression of Ezh2 mRNA follows let-7 mimic transfection in immature hippocampal neurons. In contrast, expression of PSD95/Dlg4 gene mRNA, an Ezh2 target gene in these cells, was found to be significantly enhanced. $\left(n=3,{ }^{*} p<0.05\right)$.

\section{Discussion}

During early stages of hippocampal development, the repressive activity of the PRC2 complex is critical for both silencing the expression of non-neuronal gene programs (e.g., osteogenic lineage genes) and repressing the expression of neuronal genes coding key synaptic components (e.g., PSD95/Dlg4) that are mainly required at later stages of hippocampal maturation [6,7]. Previous work from our group demonstrated that Ezh2, the catalytic subunit of PRC2, was differentially expressed during maturation of hippocampal neurons; in immature neurons, Ezh2 was abundantly expressed, whereas in mature neurons the expression of Ezh2 was significantly reduced [6,7]. Here, we find that the microRNAs let-7 and miR-124, which are highly enriched in mature hippocampal cells (20 DIV), can mediate downregulation of Ezh2 expression in these cells. Although these data argue in favor of the hypothesis that, during hippocampal maturation, the Ezh2 repressive activity (as part of the PRC2 complex) can be controlled through a miR-mediated post-transcriptional mechanism, it is necessary to consider the contribution of additional mechanisms to this Ezh2 downregulation. These additional mechanisms include changes in histone tail modifications that can inhibit transcription at the Ezh2 gene promoter in hippocampal cells [7] and the proteasome-mediated Ezh2 protein degradation in brain cells [11]. Therefore, it is tempting to propose that Ezh2 expression is tightly controlled during hippocampal maturation by a combination of transcriptional, post-transcriptional, and post-translational mechanisms.

In agreement with the results reported here, miR-124 has been previously found upregulated during mammalian neuronal differentiation $[14,21,22]$ where it contributed to neuronal survival [27]. Moreover, miR-124 has been shown to target the Ezh2 3'-UTR in neuronal cell lines (N2a and P19), thereby downregulating Ezh2 expression and reducing PRC2-Ezh2-mediated gene silencing required for proper progression of neuronal differentiation [28]. Interestingly, miR-124 has also been proposed to be a critical regulator of PRC2-independent functions of Ezh2 during early neural lineage commitment. In this case, miR-124 targeted and downregulated the expression of USP14 [29], a deubiquitinase enzyme that binds to the Ezh2 protein, and prevented its ubiquitin-dependent degradation via proteasome [30]. Together with these previous findings, our results provide further support for the critical role of miR-124 during neuronal function, as miR-124 may control Ezh2 expression at both early and late stages of neuronal differentiation.

The let-7 family members have previously been found to be expressed at elevated concentrations in brain tissues during embryogenesis [31-34]. However, a direct role of let-7 in hippocampal neuronal differentiation or plasticity has not been reported. Here, we find that a let-7-dependent decrease in 
Ezh2 mRNA is accompanied by a significant increase in PSD95/Dlg4 mRNA, further supporting the critical role of the PRC2-Ezh2 complex during inhibition of the PSD95/Dlg4 gene transcription in immature hippocampal cells [7]. Because let-7 cannot directly target the PSD95/Dlg4 gene 3'-UTR (let-7 motif is not present at this PSD95/Dlg4 sequence), our results provide a basis for proposing that, in mature hippocampal neurons, let-7 can indirectly promote the expression of relevant hippocampal plasticity components by downregulating the expression of the epigenetic repressor PRC2-Ezh2. These results extend previous findings showing that miR-125, together with fragile $\mathrm{X}$ mental retardation protein (FMRP), represses the translation of PSD95 mRNA in hippocampal neurons at early stages (7 DIV) of maturation [46,47].

Future studies must address the specific mechanisms mediating the increased expression of both miR-124 and let-7 during hippocampal neuron maturation. It has been reported that the role of miR-124 during neuronal differentiation was tightly associated with the activity of the Repressor Element-1 Silencing Transcription Factor (REST) [30], a key repressor of neuronal genes in neural progenitors, including sequences coding for the miR-124 RNA precursors [23]. Neuronal differentiation is accompanied with a progressive inhibition of this REST-dependent repression of miR-124 transcription, which results in miR-124 accumulation and subsequent downregulation of target transcripts, including those coding for REST and its associated protein MeCP1, as both transcripts contain miR-124 binding motifs at their $3^{\prime}$-UTRs $[23,48]$. Whether this type of negative feedback regulatory loop also operates during hippocampal neuronal maturation needs to be established. In the case of let-7, studies should clarify the potential role of the Lin28A/B proteins during control of the let-7 biogenesis in hippocampal neurons [49]. In mouse hippocampal neurons, brain-derived neurotrophic factor (BDNF) has been shown to directly stimulate Lin 28 expression in a developing brain, which resulted in an inhibition of let-7 biogenesis (by preventing Dicer-mediated processing of the pre-let-7 transcript and promoting its degradation) and the concomitant increase in let-7 target transcripts, including a minor subset of mRNAs coding for proteins associated with excitatory functions [50]. As BDNF-mediated gene pathways are a relevant component in the physiological function of hippocampal neurons, the contribution of this BDNF-Lin28-miR-124 axis needs to be experimentally addressed.

The contribution of the let-7 and miR-124 motifs in the Ezh2 3'-UTR inhibitory activity appears to be selective and, likely, is related to the elevated concentration of both microRNAs in mature hippocampal cells (both among the top 10 most abundant miRs in these cells). This conclusion is supported by our results indicating that a consensus motif for miR-33, a microRNA expressed at significantly lower relative concentration than let-7 and miR-124 in these mature hippocampal neurons, does not contribute to the Ezh2 3'-UTR-dependent downregulation of the upstream luciferase reporter gene. Previous reports have extensively documented that miR-33 controlled the expression of genes associated with the metabolism of cholesterol, mainly in the liver [43]. Understanding whether this microRNA also regulates specific mRNA targets (different from Ezh2) in hippocampal neurons needs further investigation.

It also remains to be determined whether let-7 and mir-124 functionally collaborate (additively or synergistically) during downregulation of Ezh2 expression in mature hippocampal neurons. Because their specific target motifs are not overlapping at the 3'-UTR sequence of the Ezh2 gene, and because independent mutation of each of these two motifs cannot completely revert the inhibitory activity associated with of this Ezh2 $3^{\prime}$ UTR sequence, it is reasonable to hypothesize that a collaborative inhibitory mechanism involving both let-7 and miR-124 operates in physiological conditions. Moreover, the possibility that these two molecules function in concert with additional regulatory mechanisms must be addressed in future studies. 


\section{Materials and Methods}

\subsection{Primary Hippocampal Cultures}

Protocols of animal management were performed according to NIH guidelines and as approved by the Ethical and Biosafety Committees of Universidad Andres Bello (014/2013; 024/2013; 001/2018). Pregnant Sprague-Dawley rats were deeply anesthetized with $\mathrm{CO}_{2}$; hippocampal cultures were prepared from E18 pups, as described previously [7,51,52].

\subsection{MicroRNA Expression Analyses Using Microarrays}

Microarray analyses were performed using $500 \mathrm{ng}$ of miRNA, purified using a mirVana ${ }^{\mathrm{TM}}$ miRNA isolation kit (Life Technologies Inc., Ambion, Burlington, ON, Canada). For miRNA labeling, the Flashtaq ${ }^{\text {TM }}$ Biotin kit (Genisphere, Hatfield, PA, USA) was used, following the manufacturer's recommendations. The miRNAs were processed for poly-A tail addition, and then ligated with adapters for biotin labeling and subsequent recognition. Labeled miRNAs were hybridized to GeneChip ${ }^{\mathrm{TM}}$ miRNA 2.0 Array (Affymetrix, Santa Clara, CA, USA), which contained 389 probes in triplicate to detect rat miRNAs. Results were analyzed using the software Affymetrix ${ }^{\mathrm{TM}}$ miRNA QC Tool.

\subsection{MicroRNA Quantitation}

The miRNAs were extracted using a mirVana ${ }^{\mathrm{TM}}$ miRNA isolation kit (Life Technologies Inc.), according to the manufacturer's instructions. Specific cDNA for each miRNA quantified was synthesized from $10 \mathrm{ng}$ of RNA, using a TaqMan ${ }^{\mathrm{TM}}$ MicroRNA Reverse Transcription Kit (Life Technologies Inc.). Then, quantitation was performed for each miRNA using TaqMan ${ }^{\mathrm{TM}}$ MicroRNA Assays (Life Technologies Inc.), followed by real-time PCR with TaqMan ${ }^{\mathrm{TM}}$ probes.

\subsection{Plasmid Construction}

Reporter plasmids were constructed by inserting the full-length $3^{\prime}$-UTR sequence of Ezh2 (NM_001107051.1) from Rattus norvegicus (between Spe I and Hind III sites), downstream of the firefly luciferase gene in the pMIR-report ${ }^{\mathrm{TM}}$ vector (Promega, Madison, WI, USA). Ezh2 3'-UTR is $407 \mathrm{bp}$ in length. Mutations were carried out by changing only two nucleotides of each MRE at the $3^{\prime}$-UTR, to prevent broad changes in the $3^{\prime}$-UTR secondary structure. Nucleotide changes were analyzed by using the Mfold (RNA-folding form) web server (http://mfold.rit.albany.edu/?q=mfold/) [53]. The $\Delta G$ values for the interactions between each miRNA and MREs were calculated using the RNAhybrid server [54]. Details of the mutations of the MREs generated for each miRNA, are shown in Table 3.

Table 3. Mutations performed at miR-124, let-7, and miR-33 motifs within the Ezh2 3'-UTR region. Specific mutated nucleotides for each miRNA recognition element (MRE) are indicated in bold black. Changes in the predicted $\Delta \mathrm{G}$ values for both wild-type (wt) and mutated miR motifs are also included.

\begin{tabular}{|c|c|c|c|c|}
\hline miR Analyzed & Ezh2 3'UTR & MRE-miR Alignments Predicted & $\Delta G$ MRE WT & $\Delta G$ MRE MUT \\
\hline WT & \multirow{3}{*}{$7-13$} & $5^{\prime}$ AAUCUACUACCUCU $3^{\prime}$ & \multirow{3}{*}{-20.1} & \multirow{3}{*}{-15.4} \\
\hline let-7 & & $3^{\prime}$ UGAUAUGUUGGAGGAUGGAGU 5' $^{\prime}$ & & \\
\hline MUT & & $5^{\prime}$ AAUCUACUACCCUU $3^{\prime}$ & & \\
\hline WT & \multirow{3}{*}{$33-39$} & $5^{\prime}$ CUCCUCUCAAACAGCUGCCUUAG $3^{\prime}$ & \multirow{3}{*}{-19.9} & \multirow{3}{*}{-15.8} \\
\hline miR-124 & & $3^{\prime}$ CCGUAAGUGGCGC-ACGGAAU 5' & & \\
\hline MUT & & $5^{\prime}$ CUCCUCUCAAACAGCUGCCUAUG $\mathbf{3}^{\prime}$ & & \\
\hline WT & \multirow{3}{*}{$195-216$} & 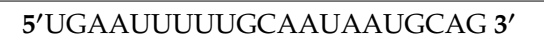 & \multirow{3}{*}{-18.2} & \multirow{3}{*}{-14.2} \\
\hline miR-33 & & $3^{\prime}$ ACGUUACGUUGAUGUUACGUG $5^{\prime}$ & & \\
\hline MUT & & 5’UGAAUUUUUGCAAUAAUGACG 3' & & \\
\hline
\end{tabular}

\subsection{Transient Transfections}

Functional 3'-UTR analyses were carried out in cultures of primary rat hippocampal neurons obtained from 18-day-old embryos (E18), that were maintained in Neurobasal ${ }^{\mathrm{TM}}$ growth medium 
(GIBCO, Gaithersburg, MD, USA) supplemented with B27 (GIBCO), 2 mM L-glutamine, 100U/mL penicillin, and $100 \mu \mathrm{g} / \mathrm{mL}$ streptomycin. Transfection was performed at 4 days in vitro (4 DIV) using Lipofectamine ${ }^{\mathrm{TM}} 3000$, following the manufacturer's instructions (Invitrogen, Carlsbad, CA, USA), and then reporter activity was evaluated at 5 DIV. To evaluate cultures at day 20 DIV, transfection with Neuromag ${ }^{\text {TM }}$ was performed at 19 DIV, according to the manufacturer's instructions (OZ Biosciences, San Diego, CA, USA). Neuronal cultures were transfected with $20 \mathrm{ng}$ of plasmid pMIR-report ${ }^{\mathrm{TM}}$ containing the $3^{\prime}$-UTR of the rat Ezh2 gene. Cultures were co-transfected with 10 pg SV-40 pRL Renilla Luciferase control reporter vector, used as a transfection control. These plasmid quantities were selected after performing dose-response curves.

\subsection{Lentivirus Production and Infection of Hippocampal Neurons}

HEK293FT cells were transfected using Lipofectamine ${ }^{\mathrm{TM}} 3000$ reagent (Invitrogen), following the manufacturer's instructions, with the pVSVg, $\mathrm{p} \Delta 8.9$, and pLKO.1-shRNA plasmids (at a 1:2:3 ratio, respectively) and a total DNA of $10 \mu \mathrm{g}$. TRC1.5 pLKO.1-puro non-mammalian shRNA control plasmid DNA (SHC002, Sigma) was used as negative control. After $12 \mathrm{~h}$, the medium was replaced and cells were maintained at $32{ }^{\circ} \mathrm{C}$ for $48 \mathrm{~h}$. Supernatants containing lentiviral particles were collected, filtered through a PVDF filter $(0.45 \mu \mathrm{m}$ pore size), and concentrated by centrifugation at $3800 \times g$ for $30 \mathrm{~min}$ at $4{ }^{\circ} \mathrm{C}$ in an Amicon ${ }^{\mathrm{TM}}$ Ultra-15 centrifugal filter (100K, Merck Millipore, Burlington, MA, USA), according to the manufacturer's instructions. Aliquots of concentrated viral particles were stored at $-80{ }^{\circ} \mathrm{C}$. Hippocampal cultures evaluated at 20 DIV were infected at 16 DIV with $1.0 \times 10^{7}$ infectious units of virus (IFU)/cell culture plate $(30 \mathrm{~mm})$ containing shRNA-Dicer or shRNA-GFP control.

\subsection{Reverse Transcriptase and Real-Time Quantitative PCR (RT-qPCR)}

Total RNA was extracted with TRIzol ${ }^{\mathrm{TM}}$ (Invitrogen), according to the manufacturer's protocol. Then, $2 \mu \mathrm{g}$ of each sample were used for reverse transcription. The qPCR was performed using SYBR Green ${ }^{\mathrm{TM}}$ PCR Master Mix (Applied Biosystems, Life Technologies Inc.). Data are presented as relative mRNA levels of the gene of interest normalized to GAPDH mRNA levels. Primers used were the following: Dicer Fw: GGCAGGTGTACTATCCGATGA, Rev: TGGTTCCATCTCAAGCAATTC; Ezh2 Fw: GCCAGACTGGGAAGAAATCTG, Rev: TCACTGGTGACTGAACACTCC; GAPDH Fw: CATGGCCTTCCGTGTTCCTA, Rev: CCTGCTTCACCACCTTCTTGAT.

\subsection{Luciferase Reporter Assay}

Luciferase activity was measured $24 \mathrm{~h}$ after transfection using a Dual-Glo ${ }^{\mathrm{TM}}$ Luciferase Assay System (Promega) in a GloMax ${ }^{\mathrm{TM}}$ 20/20 Luminometer (Promega, Turner Biosystems, Madison, WI, USA). Firefly luciferase activity was normalized to Renilla luciferase activity to minimize variations in transfection efficiency between experiments. Experiments were performed in triplicate. Quantitative data of the reporter gene assay are presented as mean $\pm \operatorname{SEM}(n=3)$.

\subsection{MicroRNA Target Prediction}

To identify miRNAs that potentially target the 3'-UTR of Ezh2 mRNAs, we used the following miRNA target prediction sites: TargetScan (http://www.targetscan.org/) [55] and miRDB (http://mirdb. org/) [56].

\subsection{Expression of Let-7 Mimics}

For the in vitro transfection of immature hippocampal neurons, the transfection mix was prepared using 90 pmol of let-7 mimic (mirVana ${ }^{\mathrm{TM}}$ miRNA mimic, Life Technologies) and $15 \mu \mathrm{L}$ of Lipofectamine ${ }^{\mathrm{TM}}$ RNAiMax (Invitrogen), according to the manufacturer's protocol, for $48 \mathrm{~h}$. The transfection was confirmed by RT-qPCR. Each experiment was repeated at least 3 times. 


\subsection{Statistical Analysis}

For the expression analysis we used Student's t-test to determine significant differences between experiments. In all figures, error bars represent the mean \pm standard error of the mean; ${ }^{*} p<0.05$, ** $p<0.01$, and ${ }^{* * *} p<0.001$.

Supplementary Materials: The following are available online at http://www.mdpi.com/1422-0067/21/22/8472/s1.

Author Contributions: L.G. performed most of the experimental work and data analyses; R.A. and F.J.B. carried out the transcriptomic studies and contributed to data analyses; G.N. and R.A.G. performed the transcriptomic studies, including the bioinformatics analyses; B.v.Z. and M.M. designed the experimental strategy, oversaw the experimental work, and wrote most parts of the manuscript. All authors have read and agreed to the published version of the manuscript.

Funding: This research was supported by FONDAP 15090007 (to R.G. and M.M.), FONDECYT 3140418 (to L.G.), FONDECYT 11190998 (to G.N.), FONDECYT 1170878 (to M.M.), FONDECYT 1181645 (to B.v.Z.), CONICYT PIA/BASAL AFB 170005 CARE UC (to B.v.Z.), and Nucleus UNAB DI-4-17/N (to B.v.Z.).

Conflicts of Interest: The authors declare no conflict of interests.

\section{Abbreviations}

$\begin{array}{ll}\text { miRs } & \text { MicroRNAs } \\ \text { CNS } & \text { Central nervous system } \\ \text { IFU } & \text { Infectious units of virus } \\ \text { FMRP } & \text { Fragile } X \text { mental retardation protein } \\ \text { BDNF } & \text { Brain-derived neurotrophic factor }\end{array}$

\section{References}

1. Gräff, J.; Kim, D.; Dobbin, M.M.; Tsai, L.-H. Epigenetic regulation of gene expression in physiological and pathological brain processes. Physiol. Rev. 2011, 91, 603-649. [CrossRef]

2. Sweatt, J.D. The emerging field of neuroepigenetics. Neuron 2013, 80, 624-632. [CrossRef]

3. Allis, C.D.; Jenuwein, T. The molecular hallmarks of epigenetic control. Nat. Rev. Genet. 2016, 17, 487-500. [CrossRef] [PubMed]

4. Margueron, R.; Reinberg, D. The Polycomb complex PRC2 and its mark in life. Nature 2011, 469, 343-349. [CrossRef] [PubMed]

5. Yu, J.-R.; Lee, C.-H.; Oksuz, O.; Stafford, J.M.; Reinberg, D. PRC2 is high maintenance. Genes Dev. 2019, 33, 903-935. [CrossRef] [PubMed]

6. Aguilar, R.; Bustos, F.J.; Saez, M.; Rojas, A.; Allende, M.L.; van Wijnen, A.J.; van Zundert, B.; Montecino, M. Polycomb PRC2 complex mediates epigenetic silencing of a critical osteogenic master regulator in the hippocampus. Biochim. Biophys. Acta 2016, 1859, 1043-1055. [CrossRef] [PubMed]

7. Henriquez, B.; Bustos, F.J.; Aguilar, R.; Becerra, A.; Simon, F.; Montecino, M.; van Zundert, B. Ezh1 and Ezh2 differentially regulate PSD-95 gene transcription in developing hippocampal neurons. Mol. Cell. Neurosci. 2013, 57, 130-143. [CrossRef]

8. Pereira, J.D.; Sansom, S.N.; Smith, J.; Dobenecker, M.-W.; Tarakhovsky, A.; Livesey, F.J. Ezh2, the histone methyltransferase of $\mathrm{PRC} 2$, regulates the balance between self-renewal and differentiation in the cerebral cortex. Proc. Natl. Acad. Sci. USA 2010, 107, 15957-15962. [CrossRef]

9. Sher, F.; Boddeke, E.; Copray, S. Ezh2 expression in astrocytes induces their dedifferentiation toward neural stem cells. Cell. Reprogram. 2011, 13, 1-6. [CrossRef]

10. Shivram, H.; Le, S.V.; Iyer, V.R. MicroRNAs reinforce repression of PRC2 transcriptional targets independently and through a feed-forward regulatory network. Genome Res. 2019, 29, 184-192. [CrossRef]

11. Yu, Y.-L.; Chou, R.-H.; Shyu, W.C.; Hsieh, S.C.; Wu, C.S.; Chiang, S.Y.; Chang, W.J.; Chen, J.N.; Tseng, Y.J.; Lin, Y.H.; et al. Smurf2-mediated degradation of EZH2 enhances neuron differentiation and improves functional recovery after ischaemic stroke. EMBO Mol. Med. 2013, 5, 531-547. [CrossRef] [PubMed]

12. Zhao, C.; Sun, G.; Ye, P.; Li, S.; Shi, Y. MicroRNA let-7d regulates the TLX/microRNA-9 cascade to control neural cell fate and neurogenesis. Sci. Rep. 2013, 3, 1-7. [CrossRef] [PubMed] 
13. Ji, F.; Lv, X.; Jiao, J. The role of microRNAs in neural stem cells and neurogenesis. J. Genet Genom. 2013, 40, 61-66. [CrossRef] [PubMed]

14. Krichevsky, A.M.; King, K.S.; Donahue, C.P.; Khrapko, K.; Kosik, K.S. A microRNA array reveals extensive regulation of microRNAs during brain development. RNA 2003, 9, 1274-1281. [CrossRef]

15. Somel, M.; Liu, X.; Tang, L.; Yan, Z.; Hu, H.; Guo, S.; Jiang, X.; Zhang, X.; Xu, G.; Xie, G.; et al. MicroRNA-driven developmental remodeling in the brain distinguishes humans from other primates. PLoS Biol. 2011, 9, e1001214. [CrossRef]

16. Khudayberdiev, S.; Fiore, R.; Schratt, G. MicroRNA as modulators of neuronal responses. Commun. Integr. Biol. 2009, 2, 411-413. [CrossRef]

17. Schratt, G. microRNAs at the synapse. Nat. Rev. Neurosci. 2009, 10, 842-849. [CrossRef]

18. De Pietri Tonelli, D.; Pulvers, J.N.; Haffner, C.; Murchison, E.P.; Hannon, G.J.; Huttner, W.B. miRNAs are essential for survival and differentiation of newborn neurons but not for expansion of neural progenitors during early neurogenesis in the mouse embryonic neocortex. Development 2008, 135, 3911-3921. [CrossRef]

19. Kawase-Koga, Y.; Otaegi, G.; Sun, T. Different timings of Dicer deletion affect neurogenesis and gliogenesis in the developing mouse central nervous system. Dev. Dyn. 2009, 238, 2800-2812. [CrossRef]

20. Makeyev, E.V.; Zhang, J.; Carrasco, M.A.; Maniatis, T. The MicroRNA miR-124 promotes neuronal differentiation by triggering brain-specific alternative pre-mRNA splicing. Mol. Cell. 2007, 27, 435-448. [CrossRef]

21. Deo, M.; Yu, J.-Y.; Chung, K.-H.; Tippens, M.; Turner, D.L. Detection of mammalian microRNA expression by in situ hybridization with RNA oligonucleotides. Dev. Dyn. 2006, 235, 2538-2548. [CrossRef] [PubMed]

22. Visvanathan, J.; Lee, S.; Lee, B.; Lee, J.W.; Lee, S.-K. The microRNA miR-124 antagonizes the anti-neural REST/SCP1 pathway during embryonic CNS development. Genes Dev. 2007, 21, 744-749. [CrossRef] [PubMed]

23. Conaco, C.; Otto, S.; Han, J.-J.; Mandel, G. Reciprocal actions of REST and a microRNA promote neuronal identity. Proc. Natl. Acad. Sci. USA 2006, 103, 2422-2427. [CrossRef] [PubMed]

24. Yoo, A.S.; Sun, A.X.; Li, L.; Shcheglovitov, A.; Portmann, T.; Li, Y.; Lee-Messer, C.; Dolmetsch, R.E.; Tsien, R.W.; Crabtree, G.R. MicroRNA-mediated conversion of human fibroblasts to neurons. Nature 2011, 476, 228-231. [CrossRef] [PubMed]

25. Xue, Y.; Ouyang, K.; Huang, J.; Zhou, Y.; Ouyang, H.; Li, H.; Wang, G.; Wu, Q.; Wei, C.; Bi, Y.; et al. Direct conversion of fibroblasts to neurons by reprogramming PTB-regulated microRNA circuits. Cell 2013, 152, 82-96. [CrossRef]

26. Cheng, L.-C.; Pastrana, E.; Tavazoie, M.; Doetsch, F. miR-124 regulates adult neurogenesis in the subventricular zone stem cell niche. Nat. Neurosci. 2009, 12, 399-408. [CrossRef]

27. Sanuki, R.; Onishi, A.; Koike, C.; Muramatsu, R.; Watanabe, S.; Muranishi, Y.; Irie, S.; Uneo, S.; Koyasu, T.; Matsui, R.; et al. miR-124a is required for hippocampal axogenesis and retinal cone survival through Lhx2 suppression. Nat. Neurosci. 2011, 14, 1125-1134. [CrossRef]

28. Neo, W.H.; Yap, K.; Lee, S.H.; Looi, L.S.; Khandelia, P.; Neo, S.X.; Makeyev, E.V.; Su, I.-H. MicroRNA miR-124 controls the choice between neuronal and astrocyte differentiation by fine-tuning Ezh2 expression. J. Biol. Chem. 2014, 289, 20788-20801. [CrossRef]

29. Doeppner, T.R.; Doehring, M.; Bretschneider, E.; Zechariah, A.; Kaltwasser, B.; Müller, B.; Koch, J.C.; Bähr, M.; Hermann, D.M.; Michel, U. MicroRNA-124 protects against focal cerebral ischemia via mechanisms involving Usp14-dependent REST degradation. Acta Neuropathol. 2013, 126, 251-265. [CrossRef]

30. Lee, S.W.; Oh, Y.M.; Lu, Y.-L.; Kim, W.K.; Yoo, A.S. MicroRNAs Overcome Cell Fate Barrier by Reducing EZH2-Controlled REST Stability during Neuronal Conversion of Human Adult Fibroblasts. Dev. Cell 2018, 46, 73-84. [CrossRef]

31. Thomson, J.M.; Parker, J.; Perou, C.M.; Hammond, S.M. A custom microarray platform for analysis of microRNA gene expression. Nat. Methods 2004, 1, 47-53. [CrossRef] [PubMed]

32. Schulman, B.R.M.; Esquela-Kerscher, A.; Slack, F.J. Reciprocal expression of lin-41 and the microRNAs let-7 and mir-125 during mouse embryogenesis. Dev. Dyn. 2005, 234, 1046-1054. [CrossRef] [PubMed]

33. Thomson, J.M.; Newman, M.; Parker, J.S.; Morin-Kensicki, E.M.; Wright, T.; Hammond, S.M. Extensive post-transcriptional regulation of microRNAs and its implications for cancer. Genes Dev. 2006, 20, 2202-2207. [CrossRef] 
34. Wulczyn, F.G.; Smirnova, L.; Rybak, A.; Brandt, C.; Kwidzinski, E.; Ninnemann, O.; Strehle, M.; Seiler, A.; Schumacher, S.; Nitsch, R. Post-transcriptional regulation of the let-7 microRNA during neural cell specification. FASEB J. 2007, 21, 415-426. [CrossRef] [PubMed]

35. Schwamborn, J.C.; Berezikov, E.; Knoblich, J.A. The TRIM-NHL protein TRIM32 activates microRNAs and prevents self-renewal in mouse neural progenitors. Cell 2009, 136, 913-925. [CrossRef] [PubMed]

36. Cimadamore, F.; Amador-Arjona, A.; Chen, C.; Huang, C.-T.; Terskikh, A.V. SOX2-LIN28/let-7 pathway regulates proliferation and neurogenesis in neural precursors. Proc. Natl. Acad. Sci. USA 2013, 110, E3017-E3026. [CrossRef] [PubMed]

37. Zhao, C.; Sun, G.; Li, S.; Lang, M.-F.; Yang, S.; Li, W.; Shi, Y. MicroRNA let-7b regulates neural stem cell proliferation and differentiation by targeting nuclear receptor TLX signaling. Proc. Natl. Acad. Sci. USA 2010, 107, 1876-1881. [CrossRef]

38. Thomas, K.T.; Gross, C.; Bassell, G.J. microRNAs Sculpt Neuronal Communication in a Tight Balance That Is Lost in Neurological Disease. Front. Mol. Neurosci. 2018, 11, 455. [CrossRef]

39. Kong, D.; Heath, E.; Chen, W.; Cher, M.L.; Powell, I.; Heilbrun, L.; Li, Y.; Ali, S.; Sethi, S.; Hassan, O.; et al. Loss of let-7 up-regulates EZH2 in prostate cancer consistent with the acquisition of cancer stem cell signatures that are attenuated by BR-DIM. PLoS ONE 2012, 7, e33729. [CrossRef]

40. Bustos, F.J.; Varela-Nallar, L.; Campos, M.; Henriquez, B.; Phillips, M.; Opazo, C.; Aguayo, L.G.; Montecino, M.; Constantine-Paton, M.; Inestrosa, N.C.; et al. PSD95 suppresses dendritic arbor development in mature hippocampal neurons by occluding the clustering of NR2B-NMDA receptors. PLoS ONE 2014, 9, e94037. [CrossRef]

41. Hutvágner, G.; McLachlan, J.; Pasquinelli, A.E.; Balint, E.; Tuschl, T.; Zamore, P.D. A cellular function for the RNA-interference enzyme Dicer in the maturation of the let-7 small temporal RNA. Science 2001, 293, 834-838. [CrossRef] [PubMed]

42. Martinez, J.; Patkaniowska, A.; Urlaub, H.; Lührmann, R.; Tuschl, T. Single-stranded antisense siRNAs guide target RNA cleavage in RNAi. Cell 2002, 110, 563-574. [CrossRef]

43. Zhang, X.; Price, N.L.; Fernández-Hernando, C. Non-coding RNAs in lipid metabolism. Vascul. Pharmacol. 2019, 114, 93-102. [CrossRef] [PubMed]

44. Kuhn, D.E.; Martin, M.M.; Feldman, D.S.; Terry, A.V.; Nuovo, G.J.; Elton, T.S. Experimental validation of miRNA targets. Methods 2008, 44, 47-54. [CrossRef] [PubMed]

45. Rebustini, I.T. A Functional MicroRNA Screening Method for Organ Morphogenesis. Curr. Protoc. Cell Biol. 2017, 74, 19.19.1-19.19.17.

46. Muddashetty, R.S.; Nalavadi, V.C.; Gross, C.; Yao, X.; Xing, L.; Laur, O.; Warren, S.T.; Bassell, G.J. Reversible inhibition of PSD-95 mRNA translation by miR-125a, FMRP phosphorylation, and mGluR signaling. Mol. Cell. 2011, 42, 673-688. [CrossRef]

47. Ifrim, M.F.; Williams, K.R.; Bassell, G.J. Single-Molecule Imaging of PSD-95 mRNA Translation in Dendrites and Its Dysregulation in a Mouse Model of Fragile X Syndrome. J. Neurosci. 2015, 35, 7116-7130. [CrossRef]

48. Wu, J.; Xie, X. Comparative sequence analysis reveals an intricate network among REST, CREB and miRNA in mediating neuronal gene expression. Genome Biol. 2006, 7, R85. [CrossRef]

49. Lee, H.; Han, S.; Kwon, C.S.; Lee, D. Biogenesis and regulation of the let-7 miRNAs and their functional implications. Protein Cell 2016, 7, 100-113. [CrossRef]

50. Huang, Y.-W.A.; Ruiz, C.R.; Eyler, E.C.H.; Lin, K.; Meffert, M.K. Dual regulation of miRNA biogenesis generates target specificity in neurotrophin-induced protein synthesis. Cell 2012, 148, 933-946. [CrossRef]

51. Sepulveda, F.J.; Bustos, F.J.; Inostroza, E.; Zúñiga, F.A.; Neve, R.L.; Montecino, M.; van Zundert, B. Differential roles of NMDA Receptor Subtypes NR2A and NR2B in dendritic branch development and requirement of RasGRF1. J. Neurophysiol. 2010, 103, 1758-1770. [CrossRef] [PubMed]

52. Varela-Nallar, L.; Grabowski, C.P.; Alfaro, I.E.; Alvarez, A.R.; Inestrosa, N.C. Role of the Wnt receptor Frizzled-1 in presynaptic differentiation and function. Neural Dev. 2009, 4, 41. [CrossRef] [PubMed]

53. Zuker, M. Mfold web server for nucleic acid folding and hybridization prediction. Nucleic Acids Res. 2003, 31, 3406-3415. [CrossRef] [PubMed]

54. Rehmsmeier, M.; Steffen, P.; Hochsmann, M.; Giegerich, R. Fast and effective prediction of microRNA/target duplexes. RNA 2004, 10, 1507-1517. [CrossRef] [PubMed] 
55. Grimson, A.; Farh, K.K.-H.; Johnston, W.K.; Garrett-Engele, P.; Lim, L.P.; Bartel, D.P. MicroRNA targeting specificity in mammals: Determinants beyond seed pairing. Mol. Cell. 2007, 27, 91-105. [CrossRef] [PubMed]

56. Wong, N.; Wang, X. miRDB: An online resource for microRNA target prediction and functional annotations. Nucleic Acids Res. 2015, 43, D146-D152. [CrossRef]

Publisher's Note: MDPI stays neutral with regard to jurisdictional claims in published maps and institutional affiliations.

(C) 2020 by the authors. Licensee MDPI, Basel, Switzerland. This article is an open access article distributed under the terms and conditions of the Creative Commons Attribution (CC BY) license (http://creativecommons.org/licenses/by/4.0/). 